\title{
WEBBED SPACES, DOUBLE SEQUENCES, AND THE MACKEY CONVERGENCE CONDITION
}

\author{
ARMANDO GARCÍA-MARTÍNEZ
}

(Received 28 April 1998)

\begin{abstract}
In [3], Gilsdorf proved, for locally convex spaces, that every sequentially webbed space satisfies the Mackey convergence condition. In the more general frame of topological vector spaces, this theorem and its inverse are studied. The techniques used are double sequences and the localization theorem for webbed spaces.
\end{abstract}

Keywords and phrases. Compatible double sequence, compatible web, Mackey convergent sequence, sequential double sequence.

1991 Mathematics Subject Classification. Primary 46A16; Secondary 46A17.

1. Introduction. A web $W$ in a topological vector space $E$ is a countable family of balanced subsets of $E$, arranged in layers. The first layer of the web consists of a sequence $\left(A_{p}: p=1,2, \ldots\right)$ whose union absorbs each point of $E$. For each set $A_{p}$ of the first layer, there is a sequence $\left(A_{p q}: q=1,2, \ldots\right)$ of sets, called the sequence determined by $A_{p}$, such that

$$
\begin{gathered}
A_{p q}+A_{p q} \subset A_{p} \text { for each } q ; \\
\bigcup\left\{A_{p q}: q=1,2, \ldots\right\} \text { absorbs each point of } A_{p} .
\end{gathered}
$$

Further, layers are made up in a corresponding way such that each set of the $k$ th layer is indexed by a finite row of $k$ integers and, at each step, the above mentioned two conditions are satisfied. Suppose that one chooses a set $A_{p}$ from the first layer, then a set $A_{p q}$ of the sequence determined by $A_{p}$ and so on. The resulting sequence $S=$ $\left(A_{p}, A_{p q}, A_{p q r}, \ldots\right)$ is called a strand. Whenever we are dealing with only one strand, we can simplify the notation by writing $W_{1}=A_{p}, W_{2}=A_{p q}$, etc. Thus, $S=\left(W_{k}\right)$ is a strand, where, for each $k, W_{k}$ is a set of the $k$ th layer.

Let $S=\left(W_{k}\right)$ be a strand. Consider $x_{k} \in W_{k}$ and the series $\sum_{k=1}^{\infty} x_{k}$. The space $E$ is webbed if the series $\sum_{k=1}^{\infty} x_{k}$ is convergent for any choice of $x_{k} \in W_{k}$; and $E$ is strictly webbed if $\sum_{k=n+1}^{\infty} x_{k}$ converges to some $x \in W_{n}$ for every $n \in \mathbb{N}$ and for any choice of $x_{k} \in W_{k}$. The standard references for webs in a topological vector space are $[5,7,8]$.

Let $(E, \tau)$ be a topological vector space. $\left(x_{n}\right)_{n} \subset E$ is a Mackey null sequence if there exists a sequence of real numbers $\left(r_{n}\right)_{n}$ such that $r_{n} \rightarrow \infty$ and $r_{n} x_{n} \rightarrow 0$ in $E$. We say that $\left(x_{n}\right)_{n} \subset E$ is Mackey convergent to $x$ if $\left(x_{n}-x\right)_{n}$ is a Mackey null sequence. A topological vector space $E$ satisfies the Mackey convergence condition (M.c.c.) if every null sequence is Mackey null. 
2. Double sequences. A completing double sequence in a topological vector space $(E, \tau)$ is a family $\left(K_{j}^{n}\right)_{n, j \in \mathbb{N}}$ of balanced subsets such that

(1) $K_{j}^{n} \subset K_{j}^{n+1}$ for every $n, j$ natural numbers;

(2) $K_{j+1}^{n}+K_{j+1}^{n} \subset K_{j}^{n}$ for every $n, j$ natural numbers;

(3) $\cup_{n \in \mathbb{N}} K_{j}^{n}$ is absorbent in $E$ for every $j$ natural number;

(4) for every $j_{0} \in \mathbb{N}$, if $x_{j} \in K_{j}^{n}$ with $j>j_{0}$, then $\sum_{j=j_{0}+1}^{\infty} x_{j}$ converges in $E$ to some $x \in K_{j_{0}}^{n}$.

Moreover, $\left(K_{j}^{n}\right)_{j, n \in \mathbb{N}}$ is compatible with the topology if, for each zero neighborhood $U$ in $E$ and for every natural number $n$, there exists a natural number $J$ such that $K_{j}^{n} \subset U$ for every $j \geq J$.

For example, if $E$ is sequentially complete and has a fundamental sequence of closed bounded sets $A_{1} \subset A_{2} \subset \cdots$ such that, for each bounded set $B \subset E$, there exists $n_{0} \in \mathbb{N}$ such that $B \subset A_{n_{0}}$ (this is the case if $E$ is the strong dual of a metrizable space). In this case, we define $K_{j}^{n}=2^{-j} A_{n}$ and it is easy to verify the properties (1) to (4), above. The reader can find further information concerning double sequences in [6].

A topological vector space $(E, \tau)$, with a compatible completing double sequence $\left(K_{j}^{n}\right)$, has a Sequential Double Sequence or the SDS property if, for each $x_{m} \rightarrow 0$ in $E$, there exists $n_{0} \in \mathbb{N}$ such that, for each $j$, there exists a natural number $M_{j}$ such that $x_{m} \in K_{j}^{n_{0}}$, for every $m \geq M_{j}$.

THEOREM 1. Let $(E, \tau)$ be a topological vector space with the SDS property. Then $E$ satisfies the Mackey convergence condition.

Proof. Let $x_{m} \rightarrow 0$ in $(E, \tau)$. Let $\left(K_{j}^{n}\right)$ be a sequential double sequence, then there exists $n_{0} \in \mathbb{N}$ such that, for every $j$, there exists a natural number $M_{j}$ such that $x_{m} \in$ $K_{j}^{n_{0}}$, for every $m \geq M_{j}$. For $n, j \in \mathbb{N}$, we have $K_{j+1}^{n} \subset(1 / 2) K_{j}^{n}$; so $K_{j+2}^{n} \subset(1 / 2) K_{j+1}^{n} \subset$ $\left(1 / 2^{2}\right) K_{j}^{n}$. Consequently, for each $l \in \mathbb{N}, K_{j+1}^{n} \subset\left(1 / 2^{l}\right) K_{j}^{n}$. Note that $\left(1 / 2^{j}\right) \leq(1 / j)$, for every $j \in \mathbb{N}$ and $K_{j+j}^{n_{0}}=K_{2 j}^{n_{0}} \subset\left(1 / 2^{j}\right) K_{j}^{n_{0}} \subset(1 / j) K_{j}^{n_{0}}$. So, there exists $M_{2 j} \in \mathbb{N}$ such that $x_{m} \in K_{2 j}^{n_{0}} \subset\left(1 / 2^{j}\right) K_{j}^{n_{0}} \subset(1 / j) K_{j}^{n_{0}}$, for every $m \geq M_{2 j}$; which implies that $j x_{m} \in K_{j}^{n_{0}}$, for every $m \geq M_{2 j}$. Analogously, for $(j+1)$, there exists $M_{2(j+1)} \geq M_{2 j}$ such that $(j+1) x_{m} \in K_{j+1}^{n_{0}}$, for every $m \geq M_{2(j+1)}$; and so, for all $j \in \mathbb{N}$. Define $r_{m}=j$ if $M_{2 j} \leq m<M_{2(j+1)}$, then $\lim _{m \rightarrow \infty} r_{m}=\lim _{j \rightarrow \infty} j=\infty$. Since $\left(K_{j}^{n}\right)$ is compatible with the topology, we conclude that $r_{m} x_{m} \rightarrow 0$.

From the theorem, a space with the SDS property is a space with the Mackey convergence condition. In what follows, we study the conditions under which we have an equivalence of these two properties. First, let us introduce another type of double sequences: a topological vector space $(E, \tau)$, with a compatible completing double sequence $\left(K_{j}^{n}\right)$, has a quasi-Sequential Double Sequence or the qSDS property if, for each $x_{n} \rightarrow 0$ in $E$, there exists $n_{0}$ such that, for every $j$, there exists a natural number $M_{j}$ and a positive real number $\alpha_{j}$ such that $m>M_{j}$ implies that $x_{m} \in \alpha_{j} K_{j}^{n_{0}}$.

If $\alpha_{j}=1$, for every $j$, in a qSDS, then it becomes on SDS. So, the qSDS is more general than the SDS. The next proposition gives the condition for the equivalence.

Proposition 2. Let $(E, \tau)$ be a topological vector space with the Mackey convergence condition. Then the SDS and the qSDS are the same. 
Proof. Let $x_{m} \rightarrow 0$ in a space $(E, \tau)$ with qSDS property. By the Mackey convergence condition, there exists a scalar sequence $r_{m} \rightarrow \infty$ such that $r_{m} x_{m} \rightarrow 0$. Then there exists $n_{0}$ such that $r_{m} x_{m} \in \alpha_{j} K_{j}^{n_{0}}$, for some $\alpha_{j}>0$ whenever $m \geq M_{j}$. Hence, $x_{m} \in\left(\alpha_{j} / r_{m}\right) K_{j}^{n_{0}} \subset K_{j}^{n_{0}}$ if $m \geq M_{j}$ and $r_{m} \geq \alpha_{j}$.

Next, we see an example, where the qSDS property holds and the SDS property does not.

Let $(E,\|\cdot\|)$ be a Banach space with a sequence $\left(x_{m}\right)_{m \in \mathbb{N}}$ weakly convergent to zero and not norm convergent. Let $B$ be the closed unit ball in $E$. For each $n, j \in \mathbb{N}$, let $K_{j}^{n}=2^{-j} B$. Then $\left(K_{j}^{n}\right)$ is a compatible completing double sequence with respect to the norm topology and, consequently, with respect to any weaker topology $\tau$, especially the weak topology since the map $i:(E,\|\cdot\|) \rightarrow(E, \tau)$ is continuous. Now, $\left(x_{m}\right)_{m \in \mathbb{N}}$ is not contained in $K_{j}^{n}$, since $K_{j}^{n}$ are neighborhoods in the norm topology such that $\bigcap_{j} K_{j}^{n}=\{0\}$ and, by [4, Ex. 4] and [4, cor. of Thm. 3], $(E, \sigma)$ does not have the M.c.c. Nevertheless, $\left(x_{m}\right)_{m \in \mathbb{N}}$ is bounded with respect to both the weak and norm topologies. So, for every $K_{j}^{n}$, there exists $\alpha_{j}$ such that $\left(x_{m}\right)_{m} \subset \alpha_{j} K_{j}^{n}$.

We have the following implication: SDS $\Longrightarrow$ qSDS. This implication can be reversed if the space has the M.c.c. Furthermore SDS $\Longrightarrow$ M.c.c. So, we have the following corollary:

COROLLARY 3. Let $E$ be a topological vector space with a compatible completing double sequence. Then E has SDS property if and only if the qSDS property and M.c.c. hold.

3. Mackey convergence and sequentially webbed spaces. $E$ is sequentially webbed if it has a compatible web $W$ such that, for every null sequence $\left(x_{n}\right)_{n \in \mathbb{N}}$ in $E$, there exists a finite collection of strands $\left\{\left(W_{k}^{(1)}\right), \ldots,\left(W_{k}^{(m)}\right)\right\}$ of $W$ such that, for every natural number $k$, there exists $M_{k}$ such that $n \geq M_{k}$ implies $x_{n} \in \bigcup_{i=1}^{m} W_{k}^{(i)}$. Gilsdorf [3] proved two relations between the M.c.c. and the sequentially webbed spaces in the locally convex case.

Here, we generalize these results. One to topological vector spaces and the other to locally $r$-convex spaces. In fact, the concept of webbed spaces, introduced here, does not use local convexity. Note that in this case, in each strand, we have $2 W_{k+1} \subset$ $W_{k+1}+W_{k+1} \subset W_{k}$ so that $W_{k+1} \subset 2^{-1} W_{k}$, and then following the proof of [3, Thm. 12], we have: if $(E, \tau)$ is a sequentially webbed topological vector space, then $E$ has the M.c.c.

In order to obtain a converse of this result, we need to use a localization theorem [5, Thm. 5.6.3.].

Let $0<r \leq 1$ fixed. $A \subset E$ is $r$-convex if $\lambda A+\mu A \subset A$, for every $\lambda, \mu \geq 0$ such that $\lambda^{r}+\mu^{r}=1$. Moreover, if $A$ is balanced, we say that $A$ is absolutely $r$-convex. If $r=1$, we have the usual convexity definition.

For $U \subset E$ balanced and absorbent, let $q_{u}: E \rightarrow \mathbb{R}^{+}$be the Minkowski functional defined by $x \rightarrow \inf \{\rho>0: x \in \rho U\} . q_{u}$ is an $r$-seminorm if $q_{u}(x+y)^{r} \leq q_{u}(x)^{r}+$ $q_{u}(y)^{r}$. Furthermore, if $q_{u}^{-1}(0)=0$, it is called an $r$-norm. $(E, \tau)$ is locally $r$-convex if it has a fundamental system of zero neighborhoods formed by $r$-convex sets.

Now, we can use the $E_{B}$ spaces for locally $r$-convex spaces. $(E, \tau)$ locally $r$-convex space is locally $r$-Baire if, for every bounded set $A \subset E$, there exists $B$ absolutely $r$ convex and bounded such that $A \subset B$ and the space $\left(E_{B}, \rho_{B}\right)$ is a Baire space, where $E_{B}$ 
is the span of $B$ and $\rho_{B}$ is the topology generated by the $r$-norm $q_{B}^{r}$.

THEOREM 4. Let $(E, \tau)$ be a locally $r$-Baire locally $r$-convex space and strictly webbed. If $E$ satisfies the Mackey convergence condition, then $E$ is sequentially webbed.

Proof. Let $W$ be a strict web in $E ;\left(x_{n}\right)_{n} \subset E$ a null sequence, and $r_{n} \rightarrow \infty$ a sequence of real numbers such that $r_{n} x_{n} \rightarrow 0$ in $E$. Let $A=\left\{r_{n} x_{n}: n \in \mathbb{N}\right\}, A$ is bounded, then there exists a bounded absolutely $r$-convex set $B$ such that $\left(E_{B}, \rho_{B}\right)$ is a Baire space and $A$ is a bounded set in $E_{B}$. The identity map $i: E_{B} \rightarrow E$ is continuous. Hence, by the localization theorem, $i$ has a closed graph and there exists a strand $\left(W_{k}\right)$ such that $i^{-1}\left(W_{k}\right)=E_{B} \cap W_{k}$ is a zero neighborhood in $\left(E_{B}, \rho_{B}\right)$ for every $k$. Finally, $A \subset$ $\alpha_{k}\left(E_{B} \cap W_{k}\right) \subset \alpha_{k} W_{k}$ for some $\alpha_{k}$, a positive real number. So, $r_{n} x_{n} \in \alpha_{k} W_{k}$ and $x_{n} \in$ $\left(\alpha_{k} / r_{n}\right) W_{k} \subset W_{k}$, for $n$ sufficiently large such that $\left|\left(\alpha_{k} / r_{n}\right)\right| \leq 1$.

\section{REFERENCES}

[1] P. P. Carreras and J. Bonet, Barrelled Locally Convex Spaces, North-Holland Mathematics Studies, vol. 131, North-Holland Publishing Co., Amsterdam, New York, 1987. MR 88j:46003. Zbl 614.46001.

[2] M. De Wilde, Closed Graph Theorems and Webbed Spaces, Research Notes in Mathematics, vol. 19, Pitman (Advanced Publishing Program), Boston, Mass. London, 1978. MR 81j:46013. Zbl 373.46007.

[3] T. E. Gilsdorf, The Mackey convergence condition for spaces with webs, Internat. J. Math. Math. Sci. 11 (1988), no. 3, 473-483. MR 89f:46003. Zbl 664.46003.

[4] _ Mackey convergence and quasi-sequentially webbed spaces, Internat. J. Math. Math. Sci. 14 (1991), no. 1, 17-26. MR 92d:46012a. Zbl 765.46002.

[5] H. Jarchow, Locally Convex Spaces, B. G. Teubner, Stuttgart, 1981, Mathematical Textbooks. MR 83h:46008. Zbl 466.46001.

[6] J. Kakol, Strongly Lindelöf spaces, Baire-type property and sequential closure conditions for inductive limits of metrizable spaces, Functional analysis (Berlin) (S. Dierolf et al., eds.), de Gruyter, 1996, pp. 227-239. MR 97m:46005. Zbl 906.46003.

[7] G. Kothe, Topological Vector Spaces. II, Fundamental Principles of Mathematical Science, vol. 237, Springer-Verlag, New York, Berlin, 1979. MR 81g:46001. Zbl 417.46001.

[8] W. Robertson, On the closed graph theorem and spaces with webs, Proc. London Math. Soc. (3) 24 (1972), 692-738. MR 46 5979. Zbl 238.46005.

García-Martínez: INSTITUTO De MATEMÁticas, U.N.A.M., AREA DE LA INVESTigación CienTíficA, Circuito EXTERIOR, CiUdAd Universitaria, MÉXico, D.F. 04510

E-mail address: agarcia@gauss . math . unam.mx 


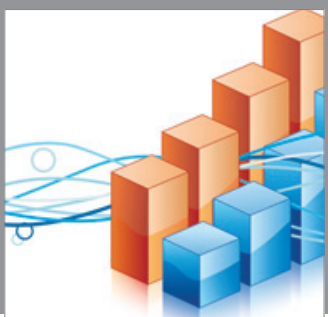

Advances in

Operations Research

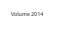

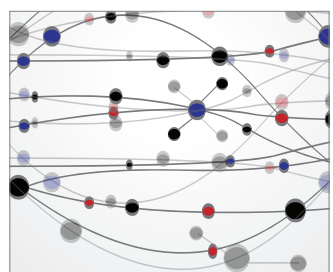

\section{The Scientific} World Journal
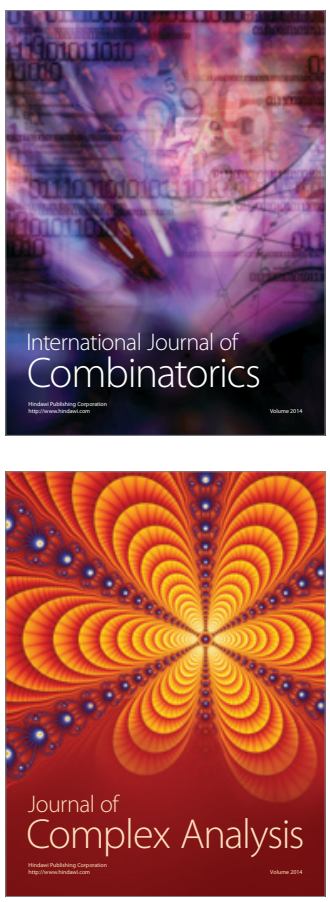

International Journal of

Mathematics and

Mathematical

Sciences
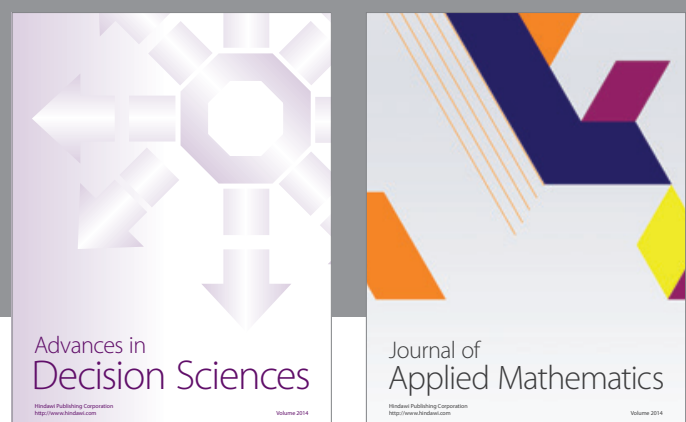

Journal of

Applied Mathematics
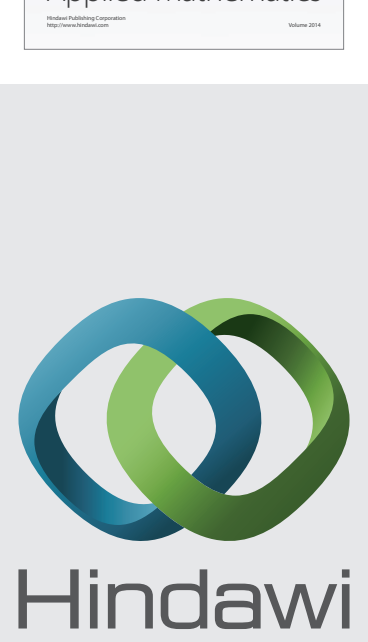

Submit your manuscripts at http://www.hindawi.com
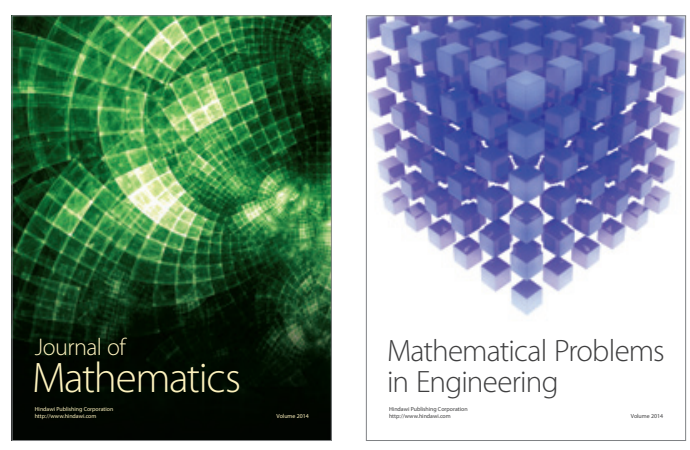

Mathematical Problems in Engineering
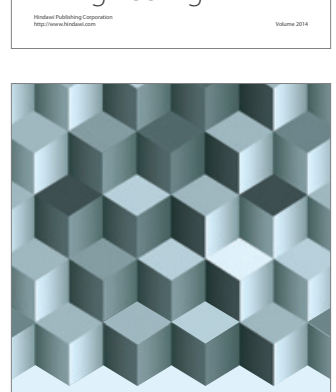

Journal of

Function Spaces
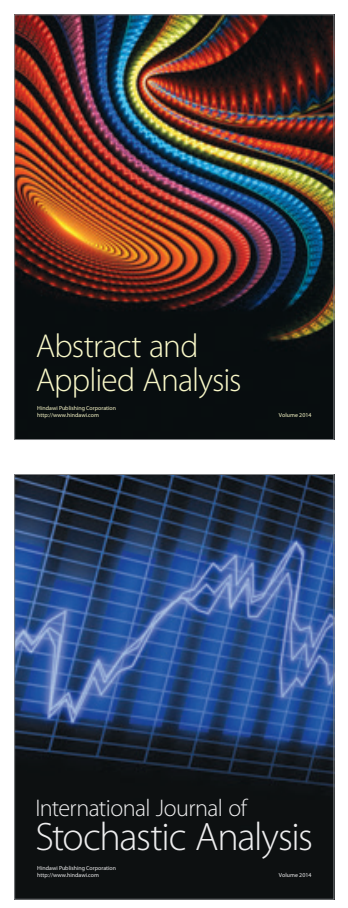

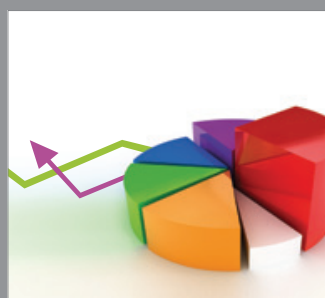

ournal of

Probability and Statistics

Promensencen
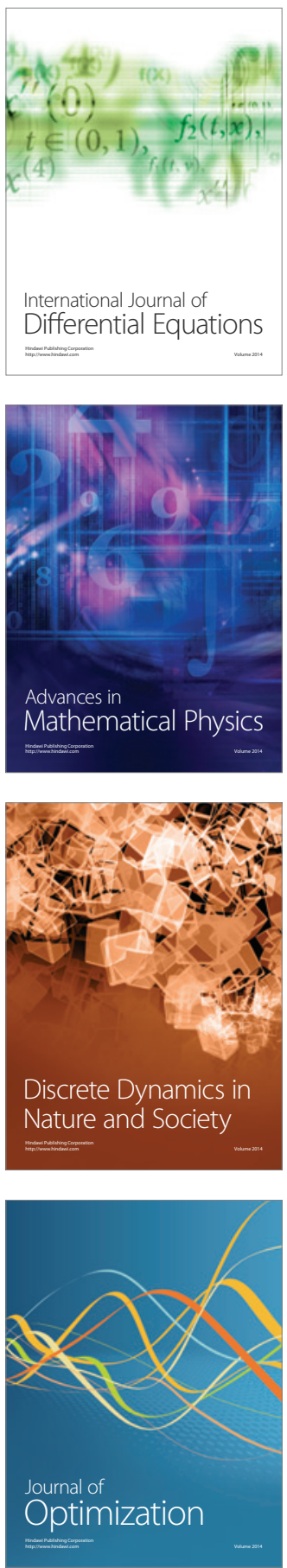